\title{
A review of Vendor Managed Inventory (VMI): from concept to processes
}

\author{
Guillaume Marquès $^{\mathrm{ab} *}$, Caroline Thierry ${ }^{\mathrm{b}}$, Jacques Lamothe ${ }^{\mathrm{a}}$ and Didier Gourc ${ }^{\mathrm{a}}$ \\ ${ }^{a}$ Université de Toulouse, Mines Albi, Centre Génie Industriel, Campus Jarlard-81013 Albi CT Cedex 09, France; \\ ${ }^{b}$ Université de Toulouse, IRIT, 118 Route de Narbonne 31062, Toulouse Cedex 09, France
}

(Final version received 10 April 2010)

\begin{abstract}
In the modern supplier-customer relationship, Vendor Managed Inventory (VMI) is used to monitor the customer's inventory replenishment. Despite the large amount of literature on the subject, it is difficult to clearly define VMI and the main associated processes. Beyond the short-term pull system inventory replenishment often studied in academic works, partners have to share their vision of the demand, their requirements and their constraints in order to fix shared objectives for the medium/long-term. In other words, the integration of VMI implies consequences for the collaborative process that links each partner's different planning processes. In this article we propose a literature review of VMI. Based on the conceptual elements extracted from this analysis, we suggest a VMI macro-process that summarises both operational and collaborative elements of VMI.
\end{abstract}

Keywords: Vendor Managed Inventory; supply chain management; collaboration

\section{Introduction}

VMI... Very Many Interpretations of Vendor Managed Inventory! The witticism emphasises the prevailing vagueness that surrounds this expression and its applications in industry. Today, using supply chain collaboration more strategically has become crucial. It enables the creation of new revenue opportunities, efficiencies and customer loyalty (Ireland and Crum 2005). Of these supply chain collaborations, Vendor Managed Inventory (VMI) is today used in industry and has inspired a large number of academic works.

However, in terms of implementation, it is clear that VMI is limited to particular situations. Nowadays VMI is almost exclusively synonymous with a distribution context. So the focus must be on how to extend distribution-VMI notions to the relationship between industrial partners.

Furthermore, describing supply chain management (SCM) Brindley (2004) underlines the difference between the notions of logistics as physical and tangible activities, and the construction and management of relationships in terms of the behavioural and intangible dimensions. Therefore, beyond the tangible short-term replenishment dimension of VMI, what does implementation of VMI mean in terms of relationships, tactics and strategic exchanges?

The purpose of this article is to explore these physical and behavioural dimensions through a review of the VMI literature. Using this review we build a global definition of the concept and the associated processes.

Thus, in Section 1 we present an overview of the literature that underlines the vagueness that surrounds VMI. In Section 2 we focus on VMI: what is VMI exactly in the literature and how VMI can be concretely implemented in the supply chain? In Section 3 we suggest a macro-process model of VMI, based on the concept. Finally, we draw several conclusions and present future research works.

\section{Syntactic literature overview}

Three main types of contribution can be found in the literature: general, case studies and models. General papers give a general definition of VMI and the main benefits of its application. Industrial case studies determine the boundaries of the VMI application, its benefits and limitations. Finally, modelling papers propose mathematical models that underline key parameters that impact VMI performance.

\subsection{Expressions used to describe VMI}

We first analysed how the term VMI is described in the literature. We are interested in the introductions and descriptive parts of the different papers. It can be noticed that authors use more than four different words or expressions to qualify VMI in the same article.

*Corresponding author. Email: marques@mines-albi.fr 
We found 26 expressions used to describe VMI (see the Appendix) which can be organised in five families:

- Concept: expressions used in a very broad and generic sense.

- Process: expressions showing a functional, process-oriented approach to VMI.

- Cooperation: expressions emphasising the relationship between partners.

- Cooperative process: this family inherits process and cooperation families.

- Technology: focuses on technologies that support VMI.

Once the families have been identified, the Appendix quantifies uses of each expression. For any given article, the figures expressed as a percentage associated with a particular expression represent the frequency of apparition of this expression in proportion to the totality of the expressions used in this article.

Globally, all authors introduce VMI in general terms belonging to the concept family. The process terms are used in a majority of papers, but are less developed. The cooperation and technology sides are mainly treated in case studies. Modelling papers broach the cooperative process, even if each author develops a particular view of the cooperative process.

This first overview of the literature underlines that a general consensus exists around the concept and the main expectations associated with VMI. However, authors have their own interpretations of the integration of the cooperative process. As Vigtil (2007a) argues, interpretations and uses of the terms are almost as numerous as the authors themselves. Consequently, the purpose of the next section is to present an overview of SCM terms and their links found in the literature.

\subsection{Other SCM terms compared to VMI}

Many terms and/or expressions relating to SCM are found in the VMI literature: VMI, Vendor Managed Replenishment (VMR), Co-Managed Inventory (CMI), Supplier-Managed Inventory (SMI), Efficient Consumer Response (ECR), Quick Response (QR), Continuous Replenishment (CR) also named Continuous Replenishment Processes (CRP) or Automatic Replenishment (AR), Consignment Inventory or Stock (CS), Just-In-Time (JIT), Retailer-Supplier Relationship (RSP), Retailer Managed Inventory (RMI), Information Sharing (IS) or Technology, etc. However, authors do not place the same interpretations on the terms. Table 1 shows the difficulty of extracting a consensus about the place of
VMI in SCM. However, common interpretations between authors can be highlighted:

- Authors who do differentiate between the terms and consider VMI as a supply chain strategy like Collaborative Planning, Forecasting and Replenishment (CPFR), Capacity Constraining Resource (CCR), QR, etc.

- Authors who consider VMI to be an element of ECR.

- Authors who see the VMI as a type of CR and who compare it to traditional inventory management. We can see that this group is exclusively composed of modelling papers.

- Authors who distinguish between VMI and CPFR. This group is close to the first one. The comparisons, however, are more precise.

- Authors who associate VMI with transfer of property (consignment).

- Authors who consider VMI as an alternative to traditional CR.

Vigtil (2007a) proposes a different way of underlining this diversity in the interpretations. She built six umbrella terms based on her literature review: RSP, AR Program, CMI, Centralised Inventory control, VMI, ECR. We can establish some links between the two classification approaches, mainly between:

- her VMI and our SCS;

- her ECR and our ECR;

- her AR Program and our CR.

This first syntactic analysis shows the difficulties in accurately defining VMI. However, even if each author uses their own words and expressions, most of them share the same concept of VMI. The next section presents the elements of this concept that we found in the literature.

\section{The concept}

Two types of element can be distinguished in general, case studies and modelling papers when seeking to identify the concept of VMI: on the one hand, the main objectives associated with VMI (Section 3.1); on the other hand, the decision levers (Section 3.2) used to reach these objectives, that we call the determinants. Moreover, Sections 3.3 and 3.4 aim at measuring determinants effect on objectives and to underline particular elements of the VMI context which are studied in the contribution of modelling (Section 3.3) and case study papers (Section 3.4). 
Table 1. VMI and other SCM terms in the literature.

\begin{tabular}{|c|c|c|c|c|}
\hline Shared Notion & Authors & $\begin{array}{l}\text { VMI is an } \\
\text { element of }\end{array}$ & $\begin{array}{l}\text { VMI is an } \\
\text { alternative or is } \\
\text { different from }\end{array}$ & $\begin{array}{c}\text { VMI is } \\
\text { synonymous } \\
\text { with }\end{array}$ \\
\hline \multirow{3}{*}{$\begin{array}{l}\text { A supply chain } \\
\text { strategy }\end{array}$} & (Wong et al. 2009) & IS & ECR, CPFR, QR & \\
\hline & $\begin{array}{l}\text { (Disney et al. 2004), (Yao and Dresner 2008), } \\
\text { (Disney and Towill 2002b), (Disney and Towill } \\
\text { 2002a), (Disney and Towill 2003) }\end{array}$ & & $\begin{array}{l}\text { IS, CR, CPFR, } \\
\text { ECR }\end{array}$ & \\
\hline & (Yao et al. 2007a) & & CR, JIT, QR, ECR & \\
\hline \multirow{4}{*}{$\begin{array}{l}\text { An element of } \\
\text { ECR }\end{array}$} & (Holweg et al. 2005) & ECR & CPFR, CR & VMR,QR \\
\hline & (Kaipia and Tanskanen 2003), (Holmström 1998) & ECR & & \\
\hline & (Kuk 2004) & ECR, QR & & \\
\hline & (Kauremaa et al. 2007), (De Toni and Zamolo 2005) & ECR & & $\mathrm{CR}$ \\
\hline \multirow[t]{3}{*}{$\begin{array}{l}\text { An element of } \\
\text { Continuous } \\
\text { Replenishment } \\
\text { (CR) }\end{array}$} & $\begin{array}{l}\text { (Nagarajan and Rajagopalan 2008), (Yu and Liu } \\
\text { 2008), (Cai et al. 2008), (Zhu and Peng 2008), } \\
\text { (Bichescu and Fry 2009), (Mishra and Raghunathan } \\
\text { 2004) }\end{array}$ & $\mathbf{C R}$ & RMI & \\
\hline & (Vigtil and Dreyer 2008) & $\mathbf{C R}$ & CPFR & \\
\hline & (Småros et al. 2003) & IS, CR & RMI & \\
\hline \multirow{6}{*}{$\begin{array}{l}\text { An alternative to } \\
\text { CPFR }\end{array}$} & (Sari 2008) & & CPFR & $\mathrm{CR}$ \\
\hline & (Meixell and Gargeya 2005) & & CPFR & \\
\hline & $\begin{array}{l}\text { (Nachiappan et al. 2007), (Nachiappan and Jawahar } \\
\text { 2007) }\end{array}$ & IT & CPFR & \\
\hline & (Achabal et al. 2000) & & CPFR, QR & \\
\hline & (Vigtil and Dreyer 2008) & $\mathrm{CR}$ & CPFR & \\
\hline & (Holweg et al. 2005) & ECR & CPFR, CR & VMR,QR \\
\hline \multirow{3}{*}{$\begin{array}{l}\text { An alternative to } \\
\text { CR }\end{array}$} & $\begin{array}{l}\text { (Gronalt and Rauch 2008), (Lee et al. 2000), (Al- } \\
\text { Ameri et al. 2008), (Cetinkaya and Lee 2000), } \\
\text { (Song and Dinwoodie 2008) }\end{array}$ & & $\mathbf{C R}$ & \\
\hline & (Dong and $\mathrm{Xu} 2002$ ) & & $\mathbf{C R}$ & $\mathrm{CS}$ \\
\hline & (Holweg et al. 2005) & ECR & CPFR, CR & VMR,QR \\
\hline \multirow{3}{*}{$\begin{array}{l}\text { Synonymous } \\
\text { with CR }\end{array}$} & $\begin{array}{l}\text { (Clark and Hammond 1997), (Southard and } \\
\text { Swenseth 2008), (Waller et al. 1999) }\end{array}$ & & & $\mathbf{C R}$ \\
\hline & (Sari 2008) & & CPFR & $\mathbf{C R}$ \\
\hline & (Kauremaa et al. 2007), (De Toni and Zamolo 2005) & ECR & & $\mathbf{C R}$ \\
\hline \multirow{2}{*}{$\begin{array}{l}\text { Synonymous } \\
\text { with } \\
\text { Consignment }\end{array}$} & (Zavanella and Zanoni 2009) & & & CS \\
\hline & (Dong and Xu 2002) & & $\mathrm{CR}$ & CS \\
\hline \multirow{4}{*}{ Others } & (Blatherwick 1998) & & CMI & \\
\hline & (Simchi-Levi et al. 2000), (Tyan and Wee 2003) & RSP & QR & \\
\hline & $\begin{array}{l}\text { (Henningsson and Lindén 2005), (Gröning and } \\
\text { Holma 2007) }\end{array}$ & SCM & & \\
\hline & (Kaipia et al. 2002), (Dong et al. 2007) & & JIT & \\
\hline
\end{tabular}




\subsection{Objectives of $V M I$}

Expressions extracted from the concept and process families provide all the elements needed to identify the objectives of VMI. According to Tang (2006), the customer's target is to ensure higher consumer service level with lower inventory costs. The supplier's target is to reduce production, inventory and transportation costs. However, we can identify shared objectives, which permit the creation of better collaboration between partners and thus the attainment of the main objectives: tightening the different flows, speeding up the supply chain (Holweg et al. 2005) and reducing the bullwhip effect (Achabal et al. 2000, Cetinkaya and Lee 2000, Disney and Towill 2003, Holweg et al. 2005).

\subsection{VMI determinants}

Many authors focus their analysis on a single, or a limited number, of links between one objective and its associated determinants. All authors agree with the cornerstone of VMI: the transfer of customer's inventory management responsibility from customer to supplier (Kaipia and Tanskanen 2003, Kuk 2004, Holweg et al. 2005, Tang 2006, Dong et al. 2007, Gronalt and Rauch 2008).

Furthermore, implementing VMI leads the supplier to a higher replenishment frequency with smaller replenishment quantities (Dong et al. 2007, Yao et al. 2007b): from monthly replenishment to weekly, or even daily (Waller et al. 1999). As a consequence, VMI leads to greater inventory cost saving (Cetinkaya and Lee 2000) without negatively impacting the overall dynamic performance of the supply chain (Zhao and Cheng 2009). The delivery frequency appears to the supplier to be a performance lever. The supplier increases the percentage of low-cost full truckload shipments and can opt for more efficient route planning with multi stops to replenish several customers' inventories (Waller et al. 1999). The supplier gains more freedom, making decisions on quantity and timing of replenishment (Rusdiansyah and Tsao 2005). Some authors (Kauremaa et al. 2007, Claassen et al. 2008, Wong et al. 2009) translate this new degree of freedom into a better flexibility.

The supplier bases replenishment decisions on the same information as previously used by the customer to make purchase decisions (Holweg et al. 2005). So, when VMI is implemented, the supplier has a better vision of the customer's demand (Kaipia and Tanskanen 2003). This results in higher predictability (Nagarajan and Rajagopalan 2008), more accurate sales forecasting methods and more effective distribution of inventory in the supply chain (Achabal et al. 2000). According to Claassen et al. (2008), the supplier can respond to demand volatility proactively instead of reactively. Production, logistics and transportation costs can be reduced through coordinated production and replenishment plans for all customers (Tang 2006, Yu et al. 2009). Due to better visibility, the supplier is able to smooth the peaks and valleys in the flow of goods (Kaipia and Tanskanen 2003); in other words, it reduces the bullwhip effect (Zhu and Peng 2008). Disney and Towill (2003) have demonstrated that VMI can reduce this effect by $50 \%$, mainly due to the visibility of the demand via the in-transit and customer inventory levels. Yao and Dresner (2008) show that information sharing reduces the supplier's safety stock, thereby reducing the average inventory level.

As the ordering processing is changed, risk allocation changes too. Cachon (2004) explains that VMI is a particular pull contract and that in consequence the allocation of inventory risk is different from a push contract: i.e. at the supplier's inventory. As a consequence, VMI implementation most often results in a backing up of stocks from the customer to the supplier warehouse (Blatherwick 1998).

The supplier has to maintain the customer's inventory level within certain pre-specified limits (Tang 2006) based on minimum and maximum ranges (ODETTE 2004). The supplier must keep sufficient inventory at the customer's site to insure minimal customer service level (CSL). According to Yao et al. (2007b), the maximum inventory level has to be limited, otherwise the supplier will push inventory onto the customer, thereby increasing inventory costs. ODETTE (2004) emphasise the fact that minimum/ maximum inventory levels have to be mutually agreed by the partners.

Figure 1 shows the relations between VMI objectives and determinants, differentiating between individual and collaborative (supply chain) objectives. The link between one determinant and the objective is not exclusive: each objective inherits all the determinants below.

The objectives and determinants we have identified in this section constitute a consensus view of the VMI concept shared by most authors. However, we do not find a similar consensus in terms of model interpretations and applications. Furthermore, papers differ when it comes to demand structure and the nature and number of supply chain members. Consequently, the next two sections are centred on a more detailed presentation of modelling papers and case studies. 


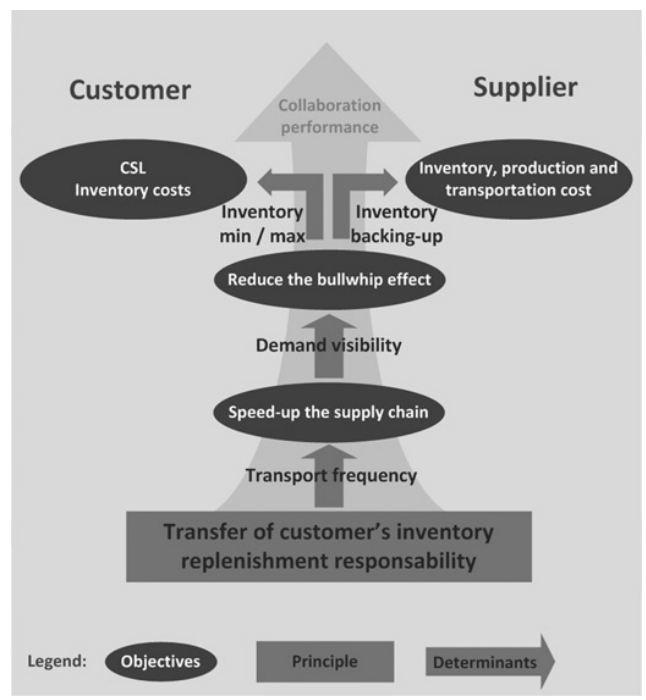

Figure 1. VMI objectives and associated determinants.

\subsection{Measuring determinant effect and VMI appli- cation benefits: modelling paper contributions}

In this section we focus on the contributions of modelling paper. These characterise the tangible short-term aspect of the VMI: the production and replenishment decision. Most are centred on short-term horizons. The main determinants considered are replenishment quantity and/or frequency decisions. These can be classified into two main model types: analytical and simulation. Both families integrate determinist and/or stochastic demand. This field of research's objectives in this category covers a broad scope. However, the problems addressed in the different contributions are mostly very specialised.

\subsubsection{Analytical models}

Analytical models represent the majority of the modelling papers. Authors are not interested in the same types of chains. These can be differentiated in two families: dyadic (two-echelons) and non-dyadic (multi-echelons) supply chains.

3.3.1.1. Dyadic supply chains. When deterministic demand is considered, the papers mostly aim at reducing inventory and most authors use Economic Order Quantity (EOQ) inventory management techniques

In the short term, Dong and $\mathrm{Xu}$ (2002) show that VMI decreases the supplier's inventory cost and increases contract purchase price under certain conditions. Yao et al. (2007b), Nagarajan and Rajagopalan (2008) and Liu et al. (2008) then show that inventory holding costs increase for the supplier and decrease for the customer, thereby emphasising the inventory backing-up in the chain with an increase in the replenishment frequency. On the other hand, Gümüs et al. (2008) determine that the association of VMI and consignment, so-called C\&VMI, could be an attractive alternative for suppliers when consignment does not decrease its total costs.

When considering stochastic demand, authors focus on the different objectives and determinants of VMI. Lee et al. (2000) and Jiang-hua and Xin (2007) analyse the benefit of information sharing and demand visibility in the chain in terms of inventory level, holding costs and total profit. Yao and Dresner (2008) study the division of benefits in the chain and show that the distribution of benefits depends on parameters such as replenishment frequency and inventory holding cost. Fry et al. (2001) suggest a $(z, Z)$ contract where the customer sets minimum $z$ and maximum $Z$ inventory levels. Suppliers pay penalties if these limits are not respected. They maximise the service level using a Markov decision process. Yao et al. (2007a) focuses on the inventory backing-up and introduce the notion of the stock-out risk that the customer wants to bear. Moreover, Song and Dinwoodie (2008), Bichescu and Fry (2009) and Zhao and Cheng (2009) propose different order quantity approaches in the face of uncertainty (demand and lead-time), studying different situations: VMI as a function of inventory levels or a function of channel power (powerful retailer, powerful supplier and equally powerful). Zhao and Cheng (2009) conclude with an aspect rarely treated in modelling papers: the value of VMI in strategic and operational terms.

3.3.1.2. Non-dyadic supply chains. In the case of non-dyadic supply chains with determinist demand, most studies work on the delivery frequency and order quantities in order to minimise different indicators: inventory holding and transportation cost, channel profit and global cost. In this context, Rusdiansyah and Tsao (2005) use the periodic Travelling Salesman Problem where one supplier replenishes $n$ customers, Nachiappan and Jawahar (2007) and Nachiappan et al. (2007) propose a genetic algorithm to solve a non-linear integer programming optimisation problem, Zavanella and Zanoni (2009) propose an optimisation model, Al-Ameri et al. (2008) propose a mixed integer-linear programme.

On the other hand, Yu et al. (2009) address the problem as the Stackelberg Game where the manufacturer is the leader. The question is to determine replenishment cycles, wholesale and retail prices in order to maximise profit. Even if the demand is determinist, it is function of the price. 
When considering stochastic demand and supply chains with one supplier and several customers, authors adopt periodic review models to determine quantity and time of replenishment orders. Consequently, they evaluate the value of new determinants in a VMI context: shipment scheduling flexibility to reduce inventory carrying costs and stock-out problems (Cetinkaya and Lee 2000); effects of transport costs and transport capacities (Yu and Liu 2008); risks shifted to the suppliers and the impact of the minimum CSL constraint on the replenishment order quantity (Wong et al. 2009); interest of products' brand substitutability on profit and actors' stock level (Mishra and Raghunathan 2004) and gains allowed by transhipment possibilities (Cai et al. 2008).

\subsubsection{Simulation models}

Discrete event simulation is used to evaluate the benefits of VMI using real demand data: Southard and Swenseth's (2008) study increased delivery frequencies and showed the reduction of inventory, delivery and stock-out costs and the improvement to the CSL. The Hewlett-Packard and Campbell Soup Company study (Waller et al. 1999) also shows that the supplier can increase its capacity utilisation using enhanced production smoothing and concludes with the relative nonimpact of demand volatility in a VMI context.

Discrete event simulation also enables comparisons between VMI and CPFR. In a four-echelon supply chain, Cigolini and Rossi (2006) evaluate service level, inventory level, inventory rotation, inventory holding cost and forecast accuracy. They conclude that VMI is justified in the case of high demand variability. In a 1-1 chain, Sari (2008) studies the order quantity in a periodic review system and evaluates CSL and total supply chain costs. In this case, CPFR has an advantage over VMI.

The impact of VMI on the bullwhip effect is also studied. Småros et al. (2003) compare traditional and VMI distributors of the same manufacturer. They show that the bullwhip effect is reduced due to the market demand visibility offered by the VMI distributors. But this benefit is more significant as the manufacturer's production planning frequency increases for products with low replenishment frequencies. In an $n-1-n$ three-echelon supply chain and a VMI EOQ re-order point system, Zhu and Peng (2008) study the decrease of the order quantity. They show that the bullwhip effect and holding inventory costs are reduced. But the profit gains are mainly for the customer, which justifies profit sharing.

Disney and Towill's research works represent the reference in terms of continuous simulation (system dynamics) of VMI. In their papers they adapt a model based on an order-up-to level called Automatic Pipeline Inventory and Order-Based Production Control System (APIOBPCS) in order to analyse VMI. Disney and Towill (2002a) make a study of system stability. Disney and Towill (2002b) also propose a Decision Support System to design VMI parameters that maximise CSL and minimise the bullwhip effect. Disney and Towill (2003) compare normal APIOBPCS and VMI-APIOBPCS to show that VMI considerably reduces the bullwhip effect. Wilson (2007) uses the APIOBCPS model to show that transport disruptions in a five-echelon supply chain are less severe with VMI.

More generally, Disney et al. (2004) propose assessing the impact of Information and Communication Technologies (ICT) using a Beer Game approach.

\subsection{VMI case studies}

This section focuses on the analysis of the case study papers. These papers underline the fact that VMI is more than an operational replenishment system. First, VMI is a part of a larger collaboration partnership that includes tactical and strategic exchanges between partners. Second, these exchanges imply information technology changes.

\subsubsection{Factors for success and failure}

VMI has been widely adopted by many industries for years. The traditional VMI implementation success story is the partnership between Wal-Mart and Procter \& Gamble. Case studies allow particular success factors to be highlighted.

Trust in the partner is the most cited success factor in the case studies (Kauremaa et al. 2007, Claassen et al. 2008, Vigtil and Dreyer 2008). This is due to the volume of information exchanges implied by the VMI implementation. Existing collaborations between the two actors therefore makes this trust easier (Dong et al. 2007).

Some authors pay particular attention to medium/ long-term collaboration. Implementation in the electrical sector shows that it allows supplier's production capacity to be scaled and a determination to be made of customer minimum and maximum inventory levels (De Toni and Zamolo 2005). According to Achabal et al. (2000), who propose VMI Decision Support System and apply it to 30 retailers, and Clark and Hammond (1997) who study the grocery industry, collaborative forecasting is the main element of this medium/long-term collaboration. Holweg et al. (2005) explain that if a supplier does not integrate several key 
items of information at the tactical planning level, the VMI impact is negative: the bullwhip effect increases.

Furthermore, the more dynamic VMI parameters such as minimum/maximum levels, the better the performance. Claassen et al. (2008), who studied five cases in different industries, and Henningsson and Lindén (2005), who studied Ikea's VMI approach, point out that dynamic arrangement for minimum and maximum inventory levels should be preferred over static ones (fixed for a year).

Other authors, however, relate cases of failure or limited improvement that provide information about failure factors. For example, in the Taiwanese grocery industry more than $50 \%$ of VMI implementations failed (Tyan and Wee 2003).

Market characteristics are often cited. Dong et al. (2007) and Tyan and Wee (2003) underline the negative consequences of a weak market competitiveness. Clark and Hammond (1997) and Deakins et al. (2008) both show that VMI is also more difficult to implement when demand is volatile or not reasonably predictable (fashions, seasonal foods, etc.).

Supply chain characteristics are also source of failure factors. According to Tyan and Wee (2003), complicated logistics flows and complex distribution channels are a reason for VMI implementation failure. Kauremaa et al. (2007) explain the adverse consequences of big distribution package size and poor choices in the composition of product assortments in different industrial sectors.

Actors' commitment is another source of failure. Actors' lack of shared understanding of the concept, and their lack of confidence in information sharing and computer systems could explain some VMI failures (Vigtil and Dreyer 2008). For example, the Ikea case study (Henningsson and Lindén 2005) showed that if demand information is not integrated in the forecast and Master Production Scheduling processes, service and inventory levels cannot be improved.

These different points imply an increasing risk of loss of control by the customer, and/or the increase of supplier's administrative costs. As far as the benefits of VMI are studied, five case studies (Claassen et al. 2008) suggest that most managers expect major cost reductions, while more benefits can be expected from improved service levels and supply chain control. In addition, Kauremaa et al. (2007) add that operational benefits of VMI are largely explained by the collaboration philosophy that characterises VMI.

The majority of papers cited above centred on industrial-distributor relationships. However, De Toni and Zamolo (2005) explain that VMI implementation at Electrolux started with a distributor, but that it was successfully developed to the other echelons in the supply chain. Gentine (2002) gives general perspectives about a VMI application to an industrial-industrial relationship: inventory levels and transport cost can be reduced using the new levels of freedom enjoyed by the supplier.

\subsubsection{Information exchange: the support technology}

One of the success factors for VMI implementation has a special place in the literature: the technological aspects. The implementation of VMI substantially increases the volume and frequency of information transmissions (Clark and Hammond 1997, De Toni and Zamolo 2005, Vigtil 2007b). Consequently, combining VMI processes and technological innovations appears as another success factor (Clark and Hammond 1997). The critical aspect is not the technology capabilities limiting the level of data exchanged, but the level of complexity (level of product variants and shipped volumes) in the set of data exchanged (Vigtil and Dreyer 2008). Furthermore, the type of information exchanged is a function of actors' production strategy (make-to-order, make-to-stock) (Vigtil 2007b). So, VMI is restricted by the actors' degrees of expertise. Nevertheless, Clark and Hammond (1997) argue that the cost of manual implementation of a VMI process exceeds the benefits. Successful implementations of VMI therefore depend on IT platforms, communication technology and product identification and tracking systems such as EDI, UCC, ERP, etc. (Waller et al. 1999).

Inaccurate demand and inventory information also affect the optimal order quantities determined by the manufacturer, thereby reducing the profits of all partners. A combination of EDI and RFID can, therefore, improve VMI efficiency and effectiveness (Yao et al. 2007a).

\subsubsection{Contributions made by trade associations}

The trade association points of view share many of the elements previously cited in the general, modelling and case study papers, but always with a certain disparity in the interpretation of words. However, the main focus of these contributions is the fundamental importance of the collaboration/agreement dimension of VMI.

VMI impacts three main processes of the SCOR model proposed by the Supply Chain Council (2008). Here, VMI is defined as 'a concept for planning and control of inventory, in which the supplier has access to the customer's inventory data and is responsible for maintaining the inventory level required by the customer. Re-supply is performed by the vendor through regularly scheduled reviews of the on-site inventory. 
The supplier takes responsibility for the operational management of the inventory within a mutually agreed framework of performance targets, which are constantly monitored and updated to create an environment of continuous improvement'.

For the VICS Association (VICS 2004), VMI is a scenario for implementing CPFR where the supplier is responsible for all steps in the replenishment process.

Several trade associations also propose XML standards for collaborative processes between industrial partners (GS1, ODETTE and Rosetta Net). All agree that with VMI processes, the supplier has access to the customer's inventory data. The supplier is responsible for generating purchase orders and maintaining the customer's inventory levels between the agreed minimum and maximum levels. ROSETTANET (2002) suggests various implementation processes: 'Specific quantities of minimum and maximum inventory target levels are communicated with each order forecast or, alternatively, may be predefined by the two trading partners and periodically reviewed for modification during the contract period.' Similarly, ODETTE (2004) distinguishes between cases where the customer validates a proposition made by its suppliers (CMI) and cases where the supplier is fully autonomous (VMI). It also proposes formulas for dynamically defining these inventory levels.

\section{Proposed VMI macro-process}

\subsection{The macro-process}

In summary, it appears that the literature examining VMI covers a very broad range, and that there is a lack of consensus about the definition of the VMI model or process. On the one hand, academic papers have developed a large quantity of mathematical models for different chains and contexts for the operational replenishment decision. On the other hand, the feedback from real applications underlines the key role of tactical and/or strategic collaboration between the partners.

We therefore propose a VMI macro-process that takes this twofold vision into account. Even if the literature usually examines VMI in a distribution context, the model proposed here is more general and allows industrial partnering to be represented.

Based on the literature review, a VMI concept can be summarised as follows: 'VMI is a replenishment pull system where the supplier is responsible for the customer's inventory replenishment, inside a collaborative pre-established medium/long-term scope'.

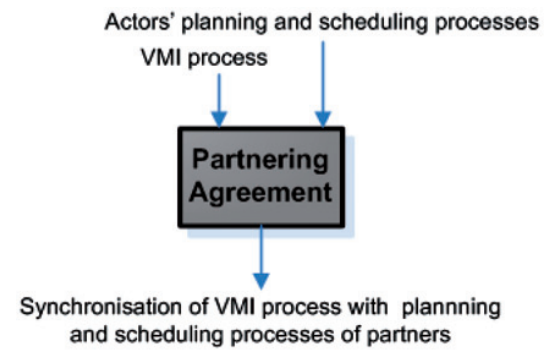

Figure 2. Partnering Agreement.

The transition from the traditional supplier-customer push relationship to a pull relationship is due to two main transformations:

- there is no longer a purchase order from customer's medium-term processes, but a short-term information about the consumption of the inventory;

- the supplier's Material Requirement Planning (MRP) function no longer issues a work order, only a target level for the supplier's inventory.

However, VMI represents more than this pull version of the traditional supplier-customer relationship. The concept states that it may lead to a situation where the partners collaborate. Thus VMI has to introduce information sharing and common decisionmaking processes.

Three processes can be defined in this VMI process:

- The Partnering Agreement (PA): specifies integration of the partners' planning processes into a VMI replenishment planning process;

- The Logistical Agreement (LA): sets the parameters used to regulate management of each article (minimum/maximum inventory level, minimum delivery quantity, transport schedule, etc.) (Gröning and Holma 2007);

- The Production and Dispatch process: monitors short-term pull decisions such as production dispatch and transport.

\subsection{Partnering Agreement}

The PA process (Figure 2) sets out the whole collaboration process. It synchronises the VMI process with each actors' planning and scheduling processes.

Many unknowns remain in terms of specifying the link when modelling the relationship. The links are created, but they have to be defined clearly. Table 2 summarises the different questions that have to be 
Table 2. Examples of link specifications for the partnering processes.

\begin{tabular}{ll}
\hline Link & Associated question (s)/choice (s) \\
\hline $\begin{array}{l}\text { Type of VMI } \\
\text { Periodicity of the LA }\end{array}$ & Which type of Production and Dispatch process? \\
& Which timescale? \\
Gross requirement expression & Which period of validity for the parameters defined by the LA? \\
Are the supplier and customer planning processes synchronised? Where are the & shared gross requirements defined? \\
Shared forecast & What is shared? \\
& What is the timescale? \\
Winimum/Maximum customer & Which period of time? \\
inventory level & How it expressed: in pieces, in days? \\
Stock information & How is it expressed: in levels, in consumption? \\
Agreed minimal transport characteristic & Periodicity: real-time, hour, day, week, etc. \\
\hline
\end{tabular}

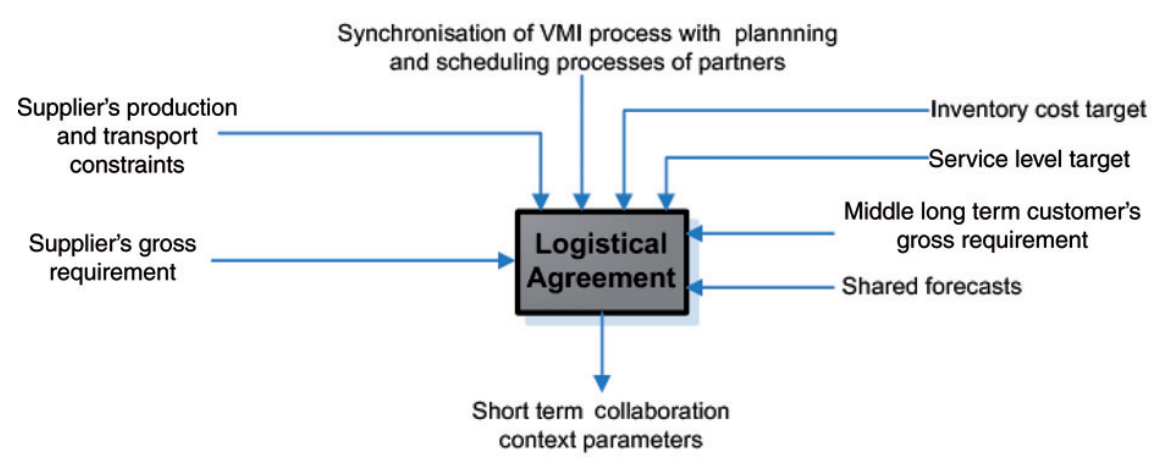

Figure 3. Logistical agreement.

answered in order to integrate the VMI process into a given collaboration process.

\subsection{Logistical Agreement}

Furthermore, trade associations and case studies stress the importance of the tactical-strategic minimum/maximum agreement. We therefore define an LA that sets these parameters, which regulate the management of each article (minimum/maximum inventory level, minimum delivery quantity, transport schedule, etc.; Gröning and Holma 2007). This allows each partner's constraints and requirements to be compared within a fixed collaborative protocol. The aim is to achieve convergent logistical parameters, which define and constrain the short-term decisions in the Production and Dispatch process.

The LA is specific to any one article. It is part of the medium/long-term decision-making process. Both supplier and customer transmit their own constraints. The customer has to ensure a minimum consumer service level and seeks to minimise inventory holding costs accordingly. The supplier, however, also has constraints, production lead times and transport capacities (lead time, frequency, lot size, etc.). They have to mutually agree on objectives and constraints for the short-term replenishment and dispatch decision-making (Figure 3).

Finally, they agree minimum and maximum customer inventory levels and transport characteristics for a pre-determined period. In order to reach the agreement, a common plan is built around shared information concerning the customer's component requirement plans and the supplier's delivery plans. Each partner includes its constraints in this plan. Two situations have been distinguished in the literature:

- either, one of the actors, usually the customer, dominates the partnership and imposes its constraints. Consequently, minima and maxima are a direct expression of these constraints. For example in Disney and Towill (2002b), the customer calculates the re-order point then passes it to the supplier;

- or, in the well-balanced partnership case the negotiation is defined by an exchange of viewpoints. It is a true collaboration in terms 
of building a plan. Dudek and Stadtler (2005) propose a process of information exchanges helping to achieve convergence between each partner's points of view.

Another important choice concerns the means used to express the targeted minimum and maximum customer inventory levels. Two different situations are described in the literature: the target is expressed in pieces or in days of stock. The choice is made according to the global industrial context and product characteristics (demand visibility, variability, nature of the product, etc.).

Furthermore, both supplier and customer can have different frequencies for their planning processes. In many industrial contexts, production and product constraints create dissimilarity between supplier and customer timescales. In this case they have to determine the appropriate LA frequency.

\subsection{The Production and Dispatch process}

The Production and Dispatch process monitors shortterm pull decisions such as production, dispatch and transport. Figures 4 and 5 distinguish between two short-term implementations according to whether production and dispatch decisions are integrated or not. These two visions are linked to the two cases we found in the literature. According to some authors, VMI has an impact on both the customer's production and dispatch decisions. Others maintain that VMI is only replenishment or a dispatch decision. The two processes are respectively called Integrated VMI and Dispatch VMI, which differ in terms of the propagation of demand uncertainty through the supply chain.

Moreover, customer demand is a fixed real quantity without VMI. With VMI, the supplier monitors the replenishment of the customer's inventory using the level of this inventory and the minimum and maximum level established by the LA. So, the dispatch process computes the net requirement expressed as an interval between a minimal and a maximal for each customer.

As far as Integrated VMI is concerned, the uncertainty is transmitted throughout the chain, first in the dispatch process then in the production process. A global short-term production and replenishment plan is made by the supplier when comparing this interval with its production constraints.

With the Dispatch VMI, the impact is less severe in terms of modifications. The choice is made within the dispatch process. The interval is transformed into a scalar at this point, independent of the production constraints. No uncertainty is transmitted to other processes.

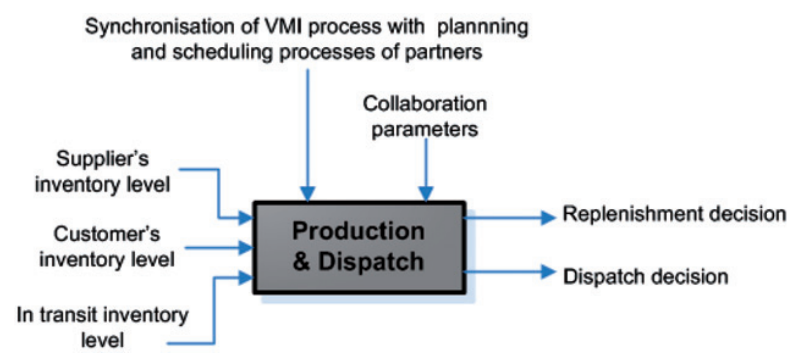

Figure 4. Production and Dispatch process in Integrated VMI.

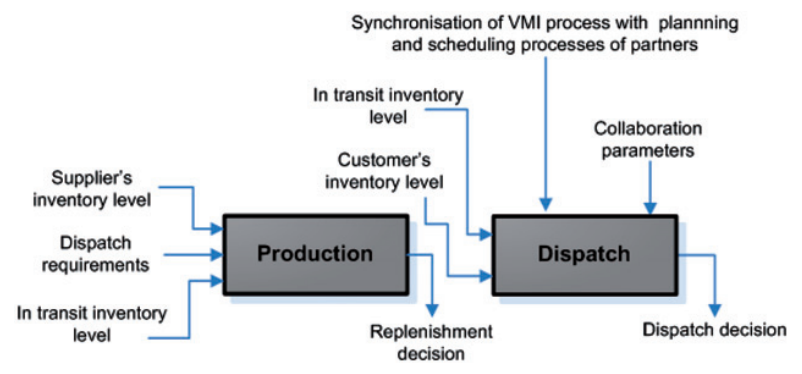

Figure 5. Production and Dispatch process in Dispatch VMI.

\subsection{Synthesis of the VMI process}

Figure 6 represents the links between the three main VMI processes. We find the different levels of decision and the distinction between the two short-term implementations, depending on whether production and dispatch decisions are integrated or not.

\section{Conclusions and future research works}

In this article we have presented an analysis of the literature on VMI. We have classified the literature into three categories: general papers, modelling papers and case studies.

In our review we first identified the concepts, objectives and decision levers considered to be associated with VMI. This enables us to propose a unified view of VMI via three main processes (PA, LA and Production and Dispatch). We emphasise the degrees of freedom available to the supplier and distinguish two types of VMI: Dispatch VMI, centred only on delivery decisions, and Integrated VMI, integrating both production and delivery decisions. All in all, most of the modelling papers look at the operational dimension of VMI: the tangible aspect proposed by Brindley (2004) and cited in the introduction. In other words, they study different implementations of the Production and Dispatch process. Case studies, on the other hand, pay particular attention to the collaborative aspect of VMI. The industrial viewpoint is mainly 


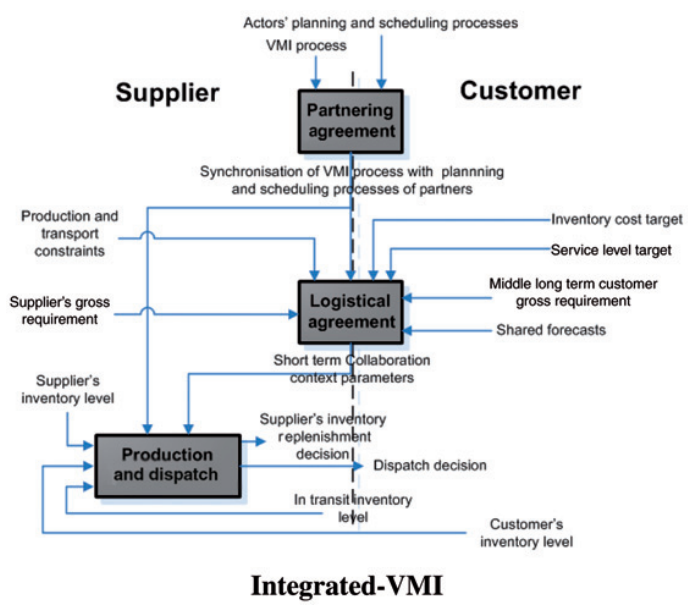

Figure 6. VMI macro-processes.

focused on the intangible and behavioural dimensions of VMI, i.e. the LA.

With the exception of Elvander et al. (2007), who propose a framework to characterise the different forms of VMI, few articles address the problem of modelling VMI by taking into account these two aspects, the operational and the collaborative. In order to define the collaboration, managers have to integrate different sources of uncertainties: evolution of the context or market, local partner behaviour, information exchange processes, etc. Our final objective is therefore to simulate the twofold dimension of VMI processes in a supply chain, and to compare their effects with traditional collaboration processes. The objective is to understand the positive and negative impacts of VMI and to identify favourable contexts.

\section{Notes on contributors}

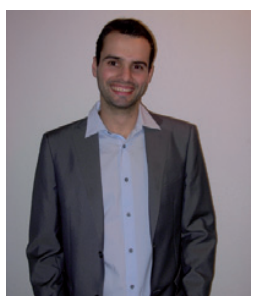

Guillaume Marquès is a $\mathrm{PhD}$ student at the Universite Toulouse/Mines Albi. $\mathrm{He}$ is a young Logistics Engineer. His works mainly focus on the risks management for SCM through a simulation approach. During his master degree he worked on project management and particularly the link with decision support systems and multi-criteria performance assessment.

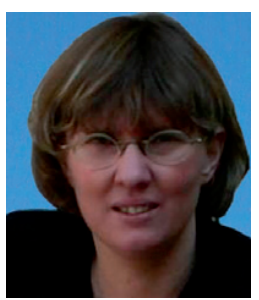

Caroline Thierry is an Associate Professor at the University of Toulouse. Her research in ONERA (French Aerospace Lab) then, since 2007, in IRIT (Institut de Recherche en Informatique de Toulouse) mostly focuses on models and decision systems in SCM.
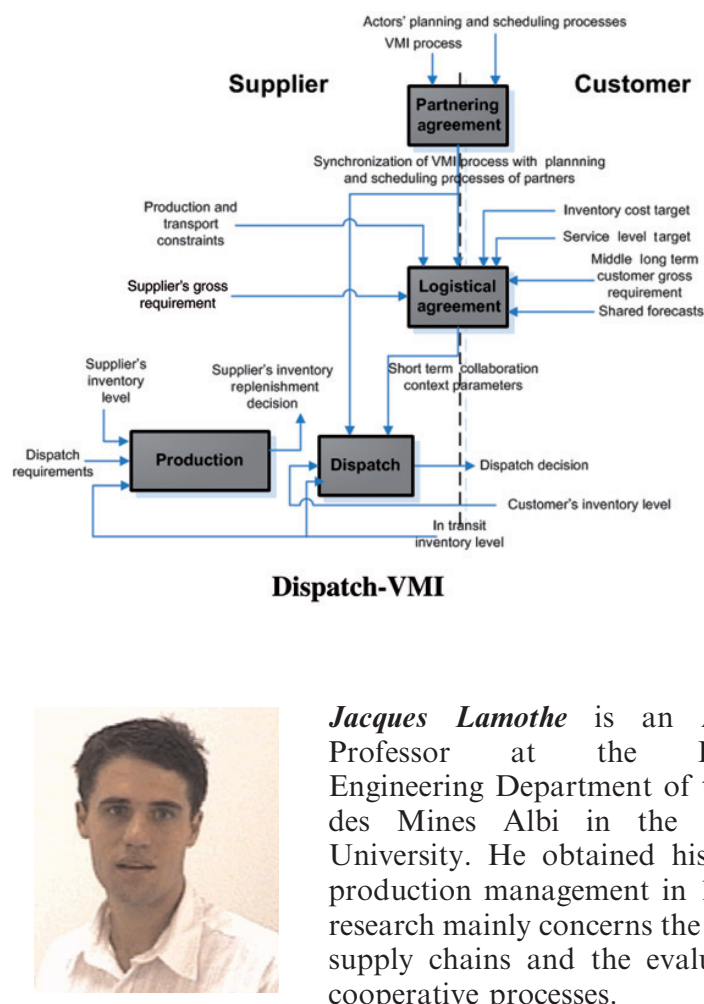

Jacques Lamothe is an Associate Professor at the Industrial Engineering Department of the Ecole des Mines Albi in the Toulouse University. He obtained his $\mathrm{PhD}$ in production management in 1996. His research mainly concerns the design of supply chains and the evaluation of cooperative processes.

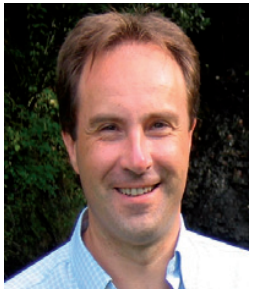

Didier Gourc, $\mathrm{PhD}$., is currently an Assistant Professor at the Ecole des Mines d'Albi-Carmaux where he teaches Project Management. He has a $\mathrm{PhD}$ from the University of Tours and he has gained industrial experiences in software development, project management and consultancy on diagnostic of production process and project management organisation since 1992. His current research interests include project management, project risk management, portfolio management and project selection. He develops his research specially in relation with pharmaceutical industry.

\section{References}

Achabal, D.D., et al., 2000. A decision support system for vendor managed inventory. Journal of Retailing, 76 (4), 430-454.

Al-Ameri, T.A., Shah, N., and Papageorgiou, L.G., 2008. Optimization of vendor-managed inventory systems in a rolling horizon framework. Computers and Industrial Engineering, 54 (4), 1019-1047.

Bichescu, B. and Fry, M., 2009. Vendor-managed inventory and the effect of channel power. OR Spectrum, 31 (1), 195-228

Blatherwick, A., 1998. Vendor-managed inventory: fashion fad or important supply chain strategy? Supply Chain Management: An International Journal, 3 (1), 10-11. 
Brindley, C., 2004. Supply chain risk. Aldershot, UK: Ashgate Publishing, Ltd.

Cachon, G.P., 2004. The allocation of inventory risk in a supply chain: push, pull, and advance-purchase discount contracts. Management Science, 50 (2), 222-238.

Cai, J.H., Wang, H.H., and Zhou, G.G., 2008. Study on a two-echelon supply chain VMI model under demand uncertainty. Proceedings of the international symposium on electronic commerce and security, 148-152.

Cetinkaya, S. and Lee, C.Y., 2000. Stock replenishment and shipment scheduling for vendor-managed inventory systems. Management Science, 46 (2), 217-232.

Cigolini, R. and Rossi, T., 2006. A note on supply risk and inventory outsourcing. Production Planning and Control, 17 (4), 424-437.

Claassen, M.J.T., Van Weele, A.J., and Van Raaij, E.M., 2008. Performance outcomes and success factors of vendor managed inventory (VMI). Supply Chain Management: An International Journal, 13 (6), 406-414.

Clark, T.H. and Hammond, J.H., 1997. Reengineering channel reordering processes to improve total supply-chain performance. Production and Operations Management, 6 (3), 248-265.

De Toni, A.F. and Zamolo, E., 2005. From a traditional replenishment system to vendor-managed inventory: a case study from the household electrical appliances sector. International Journal of Production Economics, 96 (1), 63-79.

Deakins, E., Dorling, K., and Scott, J., 2008. Determinants of successful vendor managed inventory practice in oligopoly industries. International Journal of Integrated Supply Management, 4 (3/4), 355-377.

Disney, S.M., Naim, M.M., and Potter, A., 2004. Assessing the impact of e-business on supply chain dynamics. International Journal of Production Economics, 89 (2), 109-118.

Disney, S.M. and Towill, D.R., 2002a. A discrete transfer function model to determine the dynamic stability of a vendor managed inventory supply chain. International Journal of Production Research, 40 (1), 179-204.

Disney, S.M. and Towill, D.R., 2002b. A procedure for the optimization of the dynamic response of a vendor managed inventory system. Computers and Industrial Engineering, 43 (1-2), 27-58.

Disney, S.M. and Towill, D.R., 2003. The effect of vendor managed inventory (VMI) dynamics on the bullwhip effect in supply chains. International Journal of Production Economics, 85 (2), 199-215.

Dong, Y. and Xu, K., 2002. A supply chain model of vendor managed inventory. Transportation Research Part E: Logistics and Transportation Review, 38 (2), 75-95.

Dong, Y., Xu, K., and Dresner, M., 2007. Environmental determinants of VMI adoption: an exploratory analysis. Transportation Research Part E: Logistics and Transportation Review, 43 (4), 355-369.

Dudek, G. and Stadtler, H., 2005. Negotiationbased collaborative planning between supply chains partners. European Journal of Operational Research, 163 (3), 668-687.

Elvander, M.S., Sarpola, S., and Mattsson, S.-A., 2007. Framework for characterizing the design of VMI systems. International Journal of Physical Distribution and Logistics Management, 37 (10), 782-798.

Fry, M.J., Kapuscinski, R., and Olsen, T.L., 2001. Coordinating production and delivery under a $(\mathrm{z}, \mathrm{Z})$-type vendor-managed inventory contract. Manufacturing and Service Operations Management, 3 (2), 151-173.

Gentine, P., 2002. La gestion partagée des approvisionnements peut-elle être créatrice de valeur? Accenture. Available from: http://www.accenture.com/NR/ rdonlyres/5E043862-EC58-4196-95CC-9977A34ACE3E/0/ pcmsgpai.pdf

Gronalt, M. and Rauch, P., 2008. Vendor managed inventory in wood processing industries-a case study. Silva Fennica, 42 (1), 101-114.

Gröning, A. and Holma, H., 2007. Vendor managed inventory: preparation for an implementation of a pilot project and guidance for an upcoming evaluation at Volvo Trucks in Umea. Master's thesis. Lulea University of Technology.

Gümüs, M., Jewkes, E.M., and Bookbinder, J.H., 2008. Impact of consignment inventory and vendor-managed inventory for a two-party supply chain. International Journal of Production Economics, 113 (2), 502-517.

Henningsson, E. and Lindén, T., 2005. Vendor managed inventory: enlightening benefits and negative effects of VMI for Ikea and its suppliers. Master's thesis. Lulea University of Technology.

Holmström, J., 1998. Business process innovation in the supply chain - a case study of implementing vendor managed inventory. European Journal of Purchasing and Supply Management, 4 (2-3), 127-131.

Holweg, M., et al., 2005. Supply chain collaboration: making sense of the strategy continuum. European Management Journal, 23 (2), 170-181.

Ireland, R. and Crum, C., 2005. Supply chain collaboration: how to implement CPFR and other best collaborative practices. Boca Raton, FL: J. Ross Publishing.

Jiang-hua, W. and Xin, Z., 2007. The value of centralization in a vendor-managed inventory system. 14th international conference on management science and engineering ICMSE, Beijing, 704-710.

Kaipia, R., Holmström, J., and Tanskanen, K., 2002. VMI: what are you losing if you let your customer place orders? Production Planning and Control, 13 (1), 17-25.

Kaipia, R. and Tanskanen, K., 2003. Vendor managed category management-an outsourcing solution in retailing. Journal of Purchasing and Supply Management, 9 (4), 165-175.

Kauremaa, J., Småros, J., and Holmström, J., 2007. Empirical evaluation of VMI: two ways to benefit. Proceedings of NOFOMA 2007. Available from: http:// www.tuta.hut.fi/logistics/publications/NOFOMA_2007_ Empirical_evaluation_of_VMI.pdf 
Kuk, G., 2004. Effectiveness of vendor-managed inventory in the electronics industry: determinants and outcomes. Information and Management, 41 (5), 645-654.

Lee, H.L., So, K.C., and Tang, C.S., 2000. The value of information sharing in a two-level supply chain. Management Science, 46 (5), 626-643.

Liu, J., Lu, Q., and Shi, K., 2008. Improving and evaluating various models with vendor-managed inventory. 4th IEEE international conference on management of innovation and technology ICMIT, 1395-1400.

Meixell, M.J. and Gargeya, V.B., 2005. Global supply chain design: a literature review and critique. Transportation Research Part E: Logistics and Transportation Review, 41 (6), 531-550.

Mishra, B.K. and Raghunathan, S., 2004. Retailer- vs. vendor-managed inventory and brand competition. Management Science, 50 (4), 445-457.

Nachiappan, S.P., Gunasekaran, A., and Jawahar, N., 2007. Knowledge management system for operating parameters in two-echelon VMI supply chains. International Journal of Production Research, 45 (11), 2479-2505.

Nachiappan, S.P. and Jawahar, N., 2007. A genetic algorithm for optimal operating parameters of VMI system in a two-echelon supply chain. European Journal of Operational Research, 182 (3), $1433-1452$.

Nagarajan, M. and Rajagopalan, S., 2008. Contracting under vendor managed inventory systems using holding cost subsidies. Production and Operations Management, 17 (2), 200-210.

ODETTE, 2004. Vendor managed inventory (VMI). Version 1.0.

ROSETTANET, 2002. RosettaNet collaborative forecasting process scenarios: RosettaNet collaborative forecasting-Phase (2) milestone program.

Rusdiansyah, A. and Tsao, D-B., 2005. Coordinating deliveries and inventories for a supply chain under vendor managed inventory system. JSME International Journal, Series A, Solid Mechanics and Material Engineering, 48 (2), 85-90.

Sari, K., 2008. On the benefits of CPFR and VMI: a comparative simulation study. International Journal of Production Economics, 113 (2), 575-586.

Simchi-Levi, D., Kaminsky, P., and Simchi-Levi, E., 2000. Managing the supply chain: the definitive guide for the business professional. New York: McGraw-Hill.

Småros, J., et al., 2003. The impact of increasing demand visibility on production and inventory control efficiency. International Journal of Physical Distribution and Logistics Management, 33 (4), 336-354.

Song, D.-P. and Dinwoodie, J., 2008. Quantifying the effectiveness of VMI and integrated inventory management in a supply chain with uncertain lead-times and uncertain demands. Production Planning and Control, 19 (6), 590-600.

Southard, P.B. and Swenseth, S.R., 2008. Evaluating vendormanaged inventory (VMI) in non-traditional environments using simulation. International Journal of Production Economics, 116 (2), 275-287.

Supply Chain Council, 2008. SCOR: Supply Chain Operations Reference Model. Version 9.0.

Tang, C.S., 2006. Perspectives in supply chain risk management. International Journal of Production Economics, 103 (2), 451-488.

Tyan, J. and Wee, H.M., 2003. Vendor managed inventory: a survey of the Taiwanese grocery industry. Journal of Purchasing and Supply Management, 9 (1), $11-18$.

VICS, 2004. Collaborative planning, forecasting and replenishment $\left(C P F R^{\circledR}\right)$. Available from: http://www.vics.org/ standards/CPFR_Overview_US-A4.pdf

Vigtil, A., 2007a. A framework for modelling of vendor managed inventory. PhD thesis. Norwegian University of Science and Technology.

Vigtil, A., 2007b. Information exchange in vendor managed inventory. International Journal of Physical Distribution and Logistics Management, 37 (2), 131-147.

Vigtil, A. and Dreyer, H., 2008. Critical aspects of information and communication technology in vendor managed inventory. Lean Business Systems and Beyond, IFIP International Federation for Information Processing. Vol. 257, Boston, MA: Springer, 443-451.

Waller, M., Johnson, M.E., and Davis, T., 1999. Vendor-managed inventory in the retail supply chain. Journal of Business Logistics, 20, 183-204.

Wilson, M.C., 2007. The impact of transportation disruptions on supply chain performance. Transportation Research Part E: Logistics and Transportation Review, 43 (4), 295-320.

Wong, W.K., Qi, J., and Leung, S.Y.S., 2009. Coordinating supply chains with sales rebate contracts and vendormanaged inventory. International Journal of Production Economics, 120 (1), 151-161.

Yao, Y., Dong, Y., and Dresner, M., 2007a. Analyzing information-enabled stockout management under vendormanaged inventory. Information Technology and Management, 8 (2), 133-145.

Yao, Y. and Dresner, M., 2008. The inventory value of information sharing, continuous replenishment, and vendor-managed inventory. Transportation Research Part E: Logistics and Transportation Review, 44 (3), 361-378.

Yao, Y., Evers, P.T., and Dresner, M.E., 2007b. Supply chain integration in vendor-managed inventory. Decision Support Systems, 43 (2), 663-674.

$\mathrm{Yu}$, H. and Liu, L., 2008. Replenishment and dispatch policies with transportation capacity for VMI system. International Conference on Service Systems and Service Management, Melbourne, 1-5.

Yu, Y., Chu, F., and Chen, H., 2009. A Stackelberg game and its improvement in a VMI system with a manufacturing vendor. European Journal of Operational Research, 192 (3), 929-948. 
Zavanella, L. and Zanoni, S., 2009. A one-vendor multibuyer integrated production-inventory model: the consignment stock case. International Journal of Production Economics, 118 (1), 225-232.

Zhao, Q-H. and Cheng, T.C.E., 2009. An analytical study of the modification ability of distribution centers.
European Journal of Operational Research, 194 (3), 901-910.

Zhu, Z.T. and Peng, W.Y., 2008. Simulation research on the stock control strategy of VMI combined revenue sharing. International Symposiums on Information Processing (ISIP), 657-661. 


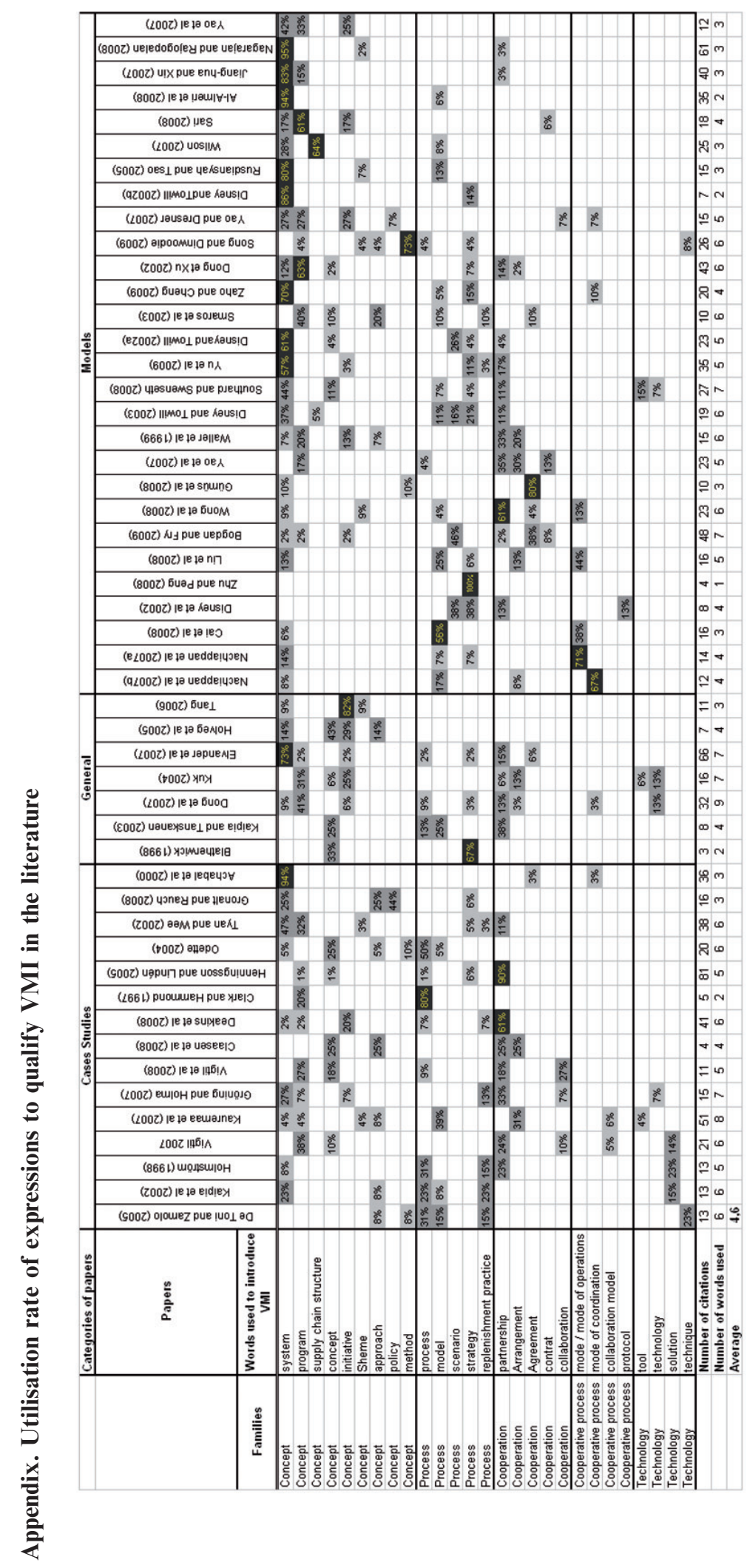

